\title{
Chitosan nanoparticles: a survey of preparation methods
}

\section{Ana Grenha*}

CBME - Centre for Molecular and Structural Biomedicine, IBB - Institute for Biotechnology and Bioengineering, University of Algarve, Campus de Gambelas, 8005-139 Faro, Portugal.

${ }^{*}$ Corresponding author:

E-mail address: amgrenha@ualg.pt

Postal address: Centre for Molecular and Structural Biomedicine (CBME), Faculty of Sciences and Technology, Building 8, Room 2.4, Campus de Gambelas, 8005-139 Faro, Portugal Phone: +351289800100 - Ext. 7441

Fax: +351289818419

Keywords: Chitosan; Desolvation; Ionic Gelation; Nanoparticles; Polyelectrolyte complexation; Preparation Methods 


\begin{abstract}
The application of macromolecules in therapy is frequently hindered by stability and/or permeation issues. These limitations have been addressed by the pharmaceutical industry through the development of suitable non-injectable drug carriers. In this context, nanoparticles have emerged as one of the most exciting tools, due to the increased surface-to-volume ratio, which provides an intimate interaction with epithelial surfaces. Nanoparticles further enable the encapsulated molecules to retain their biological activity, from the production steps to the final release. Chitosan has reached a prominent position as carrier-forming material, as diverse methods can be applied to produce nanoparticles using that excipient. These involve either hydrophilic or lipophilic environments that generally result in mild conditions or aggressive and time-consuming processes, respectively. In this review, a detailed description of methods employed to produce chitosan nanocarriers is provided, accompanied by illustrative schemes of the procedures. The emphasis is on the variables reported to affect the final properties of the vehicles.
\end{abstract}




\section{Introduction}

Many biomolecules, although therapeutically promising, are characterised by a marked instability due to inherent physicochemical and biopharmaceutical features that make their administration extremely challenging. This is the main reason why parenteral delivery is usually the unique option. Being invasive and painful, this route is not easily accepted by patients and frequently leads to therapeutic incompliance (Andrade et al. 2011). Finding adequate alternatives has become a major scientific challenge and researchers have turned their efforts towards the design of suitable drug delivery systems that enable non-invasive mucosal administration, with the hope to enhance patients' compliance and, thus, the therapeutic benefits (Zheng et al. 2010). These drug delivery carriers should exhibit characteristics that include: capacity for drug association, ability to enhance their physicochemical stability and protection of encapsulated drugs from carrier production to release. Furthermore, in many cases, carriers are expected to regulate the drug release profile, while allowing an intimate contact between molecules and mucosal barriers, contributing to their epithelial permeation (Chiellini et al. 2008, Luppi et al. 2010).

In this context, polymeric nanoparticles have appeared as promising vehicles because of several attractive properties, such as an increased surface-to-volume ratio, which offers high potential for macromolecule association (Silva et al. 2007), and the capacity to improve drug absorption. The latter either results from the reduction of epithelial resistance to drug transport, or the ability to carry the drug across the epithelium (Csaba et al. 2006, Wang et al. 2011a). Nanoparticles have also been reported to interact with mucosal epithelial membranes (de la Fuente et al. 2008a, Maurya et al. 2010), an effect that is maximal for particles of $50-500 \mathrm{~nm}$ (Jani et al. 1990, Desai et al. 1996). In addition, nanoparticles are described to permeate epithelia more easily than microparticles (Jani et al. 1990, Desai et al. 1996, Alonso 2004, Wang et al. 2011a) and, in many cases, they have been shown to provide a controlled release of the encapsulated drugs (Nagpal et al. 2010, Malam et al. 2011, Wang et al. 2011a). 
Chitosan is one of the most commonly used natural polymers in the production of nanomedicines, because it displays very attractive characteristics for drug delivery and has proved very effective when formulated in a nanoparticulate form. Properties such as its cationic character and its solubility in aqueous medium, have been reported as determinants of the success of this polysaccharide (Janes et al. 2001, Grenha et al. 2010a). However, its most attractive property relies on the ability to adhere to mucosal surfaces (Lehr et al. 1992, Blau et al. 2000, das Neves et al. 2011), leading to a prolonged residence time at drug absorption sites and enabling higher drug permeation (Takeuchi et al. 2001, Andrade et al. 2011). Chitosan has further demonstrated capacity to enhance macromolecules epithelial permeation through transient opening of epithelial tight junctions (Schipper et al. 1996, Schipper et al. 1999, Luppi et al. 2010). In addition, the polymer is known to be biocompatible and to exhibit very low toxicity (Hirano et al. 1988, Andrade et al. 2011), which are two mandatory requisites for drug delivery applications. Noticeably, chitosan has been referred to be more efficient at enhancing drug uptake when formulated in the nanoparticulate form, as compared to solution (Bowman and Leong 2006). Several recent reviews are available addressing the most prominent applications of chitosan nanoparticles, reinforcing the potential of these carriers in the biopharmaceutical and biomedical fields. Those manuscripts either focus on general drug delivery applications (Duceppe and Tabrizian 2010, Nagpal et al. 2010, Park et al. 2010, Chaudury and Das 2011, Wang et al. 2011a), delivery of genetic material (Duceppe and Tabrizian 2010, Posadas et al. 2010, Al-Qadi et al. 2011a, Tiera et al. 2011), or more specific applications, such as cancer therapy (Bisht and Maitra 2009, Tan et al. 2009, Park et al. 2010) and molecular imaging (Agrawal et al. 2010). A general message that can be taken from all these references is that materials and methods used to prepare chitosan vehicles are two major players in the carriers' efficacy (Chiellini et al. 2008).

Nevertheless, taking into account the promising applications of chitosan reported in the last two decades, in many cases related to chitosan nanoparticles, questions arise around the absence 
of commercially available chitosan-based therapeutic formulations. Some possible explanations can be found, but the approval of new excipients is known to be slow due to regulatory hurdles to demonstrate their safety for human use (Baldrick 2010). In this context, the Food and Drug Administration (FDA) has issued guidance on nonclinical studies needed to develop excipients (FDA 2005), in which excipients are considered potentially toxic substances that need to be evaluated accordingly. It is also recognised that the enormous variety of chitosan molecules, all differing in their molecular weight and deacetylation degree, not to mention in other flexible characteristics, poses many difficulties to regulatory agencies, required to perform rigorous evaluations (Kean and Tanou 2010). A very recent and complete review on chitosan safety as pharmaceutical excipient can be consulted in (Baldrick 2010).

Owing to the enormous potential demonstrated by chitosan-based nanocarriers, it seemed relevant and adequate to describe and compare in the same review, the various methods detailed in the literature for the preparation of such useful tools. In addition to providing a comprehensive report of the procedures, this review also entails a discussion on the parameters affecting the final properties of the vehicles. Many studies can be found on the application of chitosan derivatives or the use of chitosan as coating material, but these two approaches are out of the scope of the present review. Nevertheless, special emphasis will be placed on the secondary materials involved in the carriers' production.

\section{Methods for the preparation of chitosan nanoparticles}

Chitosan nanoparticles were first described in 1994, when Ohya and coworkers (1994) proposed the intravenous delivery of 5-fluorouracil, an anticancer drug, carried by chitosan nanoparticles obtained by emulsification and cross-linking. Since then, these systems have been extensively studied for drug delivery purposes and the original formulation was either used for other applications, such as the incorporation of active substances in toothpastes (Liu et al. 2007a), or modified by the application of different preparation methods (Calvo et al. 1997, Erbacher et al. 
1998, El-Shabouri 2002). In addition, many researchers have developed new formulations of chitosan nanoparticles that included secondary matrix-forming materials (Du et al. 2004, Sarmento et al. 2006a, Sarmento et al. 2006b, Grenha et al. 2010b). In particular, varied methods have been developed, mainly involving emulsification, different types of coacervation, or even slight modifications of these. More specifically, the methods include emulsion-droplet coalescence (Tokumitsu et al. 1999a), emulsion solvent diffusion (El-Shabouri 2002), reverse micellar method (Mitra et al. 2001), ionic gelation, polyelectrolyte complexation (Calvo et al. 1997, Sarmento et al. 2006a) and desolvation (Tian and Groves 1999), whose particularities will be described in the following sections. All these methods comprise bottom-up fabrication processes, which involve the assembly of molecules in solution to form defined structures (Chan and Kwok 2011), in this case, nanoparticles. Delivery systems resulting from bottom-up technologies usually display size polydispersity (Wang et al. 2011b), which in some cases limits nanoparticle usefulness. In fact, is it assumed that in a polydisperse system, larger nanoparticles might have higher drug loading capacity, while smaller nanoparticles are expected to have higher efficiency at delivering drugs to tissues or cells. This means that, even if the drug carrier has high encapsulation efficiency, the efficacy of the delivery may be poor (Fan et al. 2012), compromising the therapeutic efficacy. Interestingly, a recent technological development related to a top-down process termed particle replication in non-wetting templates (PRINT), which is a modified soft lithography technique, has demonstrated independent control over nanoparticle size, as well as other parameters that include shape, modulus (stiffness) and surface chemistry (Canelas et al. 2009, Wang et al. 2011b). Nevertheless, although this technology appears very promising in drug delivery, so far, no application was reported for chitosan, even though the authors indicate that a wide range of materials can be used, including biodegradable and biocompatible polymers (Wang et al 2011b). 
Table 1 provides summarised information on the methods applied to produce chitosan-based nanocarriers, as well as on their matrix composition, and displays a list of secondary materials that can be combined with chitosan to form the nanoparticulate systems.

\section{Emulsification and cross-linking}

As mentioned above, this method was the first to be used to form chitosan nanoparticles and involves the preparation of a W/O emulsion (this first report described the emulsification of chitosan aqueous solution in toluene, using Span $80^{\circledR}$ as emulsifier), with subsequent addition of a cross-linking agent that has the function of hardening the formed droplets. The reactive amino groups of chitosan undergo a covalent cross-linking with the aldehyde groups of glutaraldehyde (Figure 1), which is added after the emulsion formation and, consequently, after nanoparticle production (Ohya et al. 1994). Those authors pioneered the production of chitosan nanoparticles, which were used to deliver 5 -fluorouracil. Other authors used the method for the same purpose of drug delivery, but modified the oil phase composition to liquid paraffin and petroleum ether (Songjiang and Lixiang 2009). The final particle size was demonstrated to be highly dependent on stirring speed, as well as on the extent of cross-linking (Agnihotri et al. 2004, Prabaharan and Mano 2005). Several drawbacks have been progressively pointed out for this method, including the need of tedious procedures and the application of harsh cross-linking agents (Agnihotri et al. 2004). In fact, cross-linkers such as glutaraldehyde were found to cause overt toxicity and to compromise drug integrity, contributing to a progressive shift of interest towards less aggressive procedures (Janes et al. 2001). Consequently, the application of this method to obtain chitosan nanoparticles was restricted to a few works.

\section{Emulsion droplet coalescence}

This method is a derivation of the emulsification and cross-linking method described above and was first reported for microparticles preparation (Tokumitsu et al. 1999a). The same authors 
later adapted the method to prepare chitosan nanoparticles loaded with gadolinium, as a strategy for neutron-capture therapy of cancer (Tokumitsu et al. 1999b). Chitosan is dissolved in the aqueous solution of gadolinium and a small aliquot $(1 \mathrm{~mL})$ of this is added to $10 \mathrm{~mL}$ of liquid paraffin containing sorbitan sesquiolate $\left(\operatorname{Span}^{\circledR} 83\right)$. The mixture is stirred with a high-speed homogeniser, thus forming an W/O emulsion (Figure 2). In parallel, another W/O emulsion is prepared by adding $1.5 \mathrm{~mL} \mathrm{NaOH}$ to $10 \mathrm{~mL}$ of a similar outer phase. Both emulsions are then mixed using a high-speed homogeniser, leading to droplet coalescence. This results in the solidification of chitosan particles by action of $\mathrm{NaOH}$, which acts as precipitating agent. Afterwards, a further set of washing and centrifugation steps is applied using toluene, ethanol and water (Tokumitsu et al. 1999b). A similar procedure was used in a different study to encapsulate 5-fluorouracil (Anto et al. 2001). This method exploits the fact that, when two emulsions with equal outer phase are mixed together, droplets of each collide randomly and coalesce, resulting in final droplets with uniform content. The nanoparticles are formed within the emulsion-droplets (Ichikawa et al. 2006). Decreasing chitosan deacetylation degree was shown to increase particle size and to reduce nanoparticle capacity for drug association, as a consequence of the diminished capacity of ion-pair formation and de-swelling (Tokumitsu et al. 1999b). It was also found that varying chitosan concentration between 0.5 and $2.5 \%$ did not affect 5-fluorouracil encapsulation efficiency (around 70\%) or release profile, which extended over 13 hours (Anto et al. 2011). To our knowledge, no other works report the application of this method to produce chitosan nanoparticles.

\section{Emulsion solvent diffusion}

The emulsion solvent diffusion method of preparing chitosan nanoparticles is an adaptation of the original procedure developed to produce PLGA-based nanoparticles (Niwa et al. 1993), setting its basis on the partial miscibility of an organic solvent with water. The specific method for preparation of chitosan nanoparticles involves the addition of an organic phase (e.g. methylene 
chloride and acetone) containing the hydrophobic drug, to an aqueous solution containing chitosan and a stabiliser (e.g. poloxamer and lecithin), under stirring (Figure 3) (El-Shabouri 2002). This leads to the formation of an O/W emulsion which is then subjected to high pressure homogenisation. Methylene chloride is subsequently removed under reduced pressure at room temperature. At this stage, acetone diffuses to the aqueous phase, decreasing chitosan solubility and, thus, nanoparticles are formed upon polymer precipitation. An additional amount of water is usually added in order to permit the complete diffusion of acetone. Finally, nanoparticles are isolated by centrifugation.

This method demonstrated to be suitable for encapsulating hydrophobic drugs like cyclosporinA, with high encapsulation efficiencies. In spite of the limited number of studies available on the method, parameters such as chitosan molecular weight, homogenisation rate and reaction time (period of evaporation and diffusion), are expected to affect the final properties of the vehicles. The presence of acetone was also reported as essential (El-Shabouri 2002), since its rapid diffusion disturbs the organic/aqueous phase interface, which spontaneously produces a larger area and, thus, leads to the formation of much smaller droplets. Particles produced without acetone presented sizes outside the submicron range (above $1.2 \mu \mathrm{m}$ ).

Notwithstanding the ability of this method to produce effective vehicles, it is important to highlight the need for harsh preparation conditions, such as organic solvents and high shear forces, which are absent in several other methods that will be described in subsequent sections.

\section{Reverse micellisation}

The production of chitosan nanoparticles from reverse micelles was first reported by Mitra et al. (2001) as a strategy for tumour targeted delivery. Reverse micelles are W/O droplets and thus form in a W/O system (Pileni 2006), in contrast to conventional micelles that form in O/W environment. In this method of reverse micellisation, a W/O microemulsion is prepared using a lipophilic surfactant that is dissolved in an appropriate organic solvent, like $n$-hexane. 
Surfactants like sodium bis(ethyl hexyl) sulfosuccinate (AOT) or cetyl trimethylammonium bromide have been used. An aqueous phase comprising chitosan, the drug and glutaraldehyde is then added over the organic phase under continuous stirring. As depicted in Figure 4, reverse micelles are produced at this stage. Thereafter, nanoparticles are extracted following solvent evaporation (Mitra et al. 2001, Banerjee et al. 2002). It is described that an increase in the crosslinking rate results in the production of larger particles (Banerjee et al. 2002).

As compared to other emulsion-based methods, the reverse micellisation method has the advantage of producing ultrafine nanoparticles of around $100 \mathrm{~nm}$ or even less, with a narrower size range, in contrast to the larger nanocarriers (> $200 \mathrm{~nm}$ ) usually obtained by other emulsification techniques. Nevertheless, disadvantages such as the difficult isolation of nanoparticles and the need for larger amounts of solvent, have been mentioned (Tang et al. 2007).

Apart from the application in anticancer therapy, other authors have later used this method as a strategy for enzyme immobilisation (Tang et al. 2007) or to encapsulate oligonucleotides (Manchanda and Nimesh 2010).

Ionic gelation and Polyelectrolyte complexation

When chitosan has a high degree of protonation of the amine functions, it displays the capacity to form hydrogels in presence of specific polyanions. This process derives from inter- and intramolecular cross-linkages mediated by the anionic molecules (Janes et al. 2001, Terbojevich and Muzzarelli 2009) and has been used to produce chitosan-based nanoparticles by ionic gelation or polyelectrolyte complexation. It should be noted that ionic gelation is the preferred term when chitosan gelation is induced by small anionic molecules, such as phosphate, citrate, sulfate, while a polyelectrolyte complexation is considered to occur when anionic macromolecules are used instead of small molecules (Bhattarai et al. 2010). The latter approach is often referred to as complex coacervation or interfacial coacervation (Poncelet 2005, Kissel et al. 2006). 
Materials applied in the production of such chitosan nanoparticles include polymers such as dextran sulfate (Sarmento et al. 2006b), sodium alginate (Sarmento et al. 2006a, Yang et al. 2011), carrageenan (Grenha et al. 2010b), arabic gum (Avadi et al. 2010), glucomannan (Du et al. 2004), carboxymethyl cellulose (Kaihara et al. 2011), chondroitin sulfate (Yeh et al. 2011), pectin (Grabnar and Kristl 2010), heparin (Liu et al. 2007b), hyaluronic acid (de la Fuente et al. 2008b), cyclodextrins (Teijeiro-Osorio et al. 2009), poly-Y-glutamic acid (Lin et al. 2007, Lin et al. 2008) and poly(acrylic acid) (Hu et al. 2002), as well as protein-based molecules, like insulin (Bayat et al. 2008), or even DNA (Erbacher et al. 1998, Xu et al. 2011). One work reports the use of sodium lauryl sulfate (Elsayed et al. 2011). In some cases, both the aforementioned methods are combined to form nanoparticles. Examples of these systems include chitosan/alginate/tripolyphosphate (Goycoolea et al. 2009), chitosan/glucomannan/tripolyphosphate (Alonso-Sande et al. 2006), chitosan/hyaluronic acid/tripolyphosphate (de la Fuente et al. 2008b) or chitosan/cyclodextrin/tripolyphosphate (Teijeiro-Osorio et al. 2009) nanoparticles.

Benefiting from chitosan gelation in the presence of anions, Bodmeier et al. (1989) reported the production of chitosan beads for oral administration. Calvo et al. (1997) developed a very mild adaptation of ionic gelation to prepare chitosan nanoparticles in a complete hydrophilic environment. That method involves an ionic interaction between the positively charged amino groups of chitosan and the polyanion tripolyphosphate (TPP), which acts as chitosan crosslinker. Nanoparticles formation takes place immediately after the addition of a TPP solution to a solution of chitosan, under mild stirring, at room temperature (Figure 5). Stirring should be maintained for approximately 10 minutes to allow particle stabilisation and the obtained suspension centrifuged to separate the nanoparticles from unreacted chitosan and TPP. The resultant pellet of nanoparticles is then resuspended in water (Carvalho et al. 2009). As mentioned above, many different materials have been employed to produce chitosan nanoparticles by both methods. The formed nanoparticles were then used to administer different 
drugs (insulin, bovine serum albumin, polypeptidic mixtures, ovalbumin, tetanus toxoid, estradiol, carvacrol, heparin, cyclosporin-A, saponin, epidermal growth factor receptor) through various routes of administration (oral, nasal, pulmonary, ocular) (de Campos et al. 2001, Ma et al. 2002, Du et al. 2004, Cuña et al. 2006, Sarmento et al. 2007, Wang et al. 2008, Oyarzun-Ampuero et al. 2009, Azizi et al. 2010, Grenha et al. 2010b, Keawchaoon and Yoksan 2011, Rejinold et al. 2011). The author's experience in the field and other works available in the literature, have demonstrated that the final properties of chitosan nanoparticles obtained through electrostatic interaction are dependent on several parameters inherent to the preparation method. In this sense, controlling the stirring pattern (speed and type of vial, most importantly) and the conditions of centrifugation (speed and duration), is of utmost importance (Carvalho et al. 2009), independently of the specific materials composing the nanoparticle matrix. Optimising variables like materials concentration and mass ratios, as well as temperature of the reacting solutions and the volume of resuspension (Calvo et al. 1997, Carvalho et al. 2009, Fan et al. 2012), is also an important task. In fact, nanoparticles produced by polyelectrolyte complexation between two oppositely charged polymers, undergo a size increase with the incorporation of increasing amounts of one of the polymers (Hu et al. 2002, Sarmento et al. 2006b, Teijeiro-Osorio et al. 2009, Grenha et al. 2010b). In contrast, the production of nanoparticles by ionic gelation results in smaller particles for higher amounts of cross-linker (Grenha et al. 2005, Teijeiro-Osorio et al. 2009). A very recent work on chitosan/TPP nanoparticles has also established that the concentration of acetic acid used to dissolve chitosan and the temperature at which the crosslinking process occurs, strongly affect the polydispersity of the obtained nanoparticles. Interestingly, the authors demonstrated that performing the reaction below $4{ }^{\circ} \mathrm{C}$ results in a polydispersity index below 0.05 . This was attributed to the fact that reduced temperature increased hydrogen bonds between the polar groups of chitosan and the surrounding water, leading to the formation of a hydration layer around the nanoparticles, which decreased the probability of nanoparticle collision. In parallel, a concentration of $0.2 \mathrm{mg} / \mathrm{mL}$ of acetic acid was 
shown to be optimal for achieving a narrow particle size distribution, as it is the concentration required to provide sufficient protonation of chitosan for interaction with TPP (Fan et al. 2012). In addition, the type of chitosan used has also been reported as crucial. Chitosan exists under many different chemical structures, being available either as a base or a salt (different types of salts are also available, such as chloride, lactate, glutamate, etc.). Moreover, distinct molecular weights and deacetylation degrees also exist. Several studies have established a relation between these features and the final characteristics of the resultant chitosan nanoparticles $(\mathrm{Xu}$ and Du 2003, Huang et al. 2004, Zhang et al. 2004, Al-Qadi et al. 2011b), revealing that chitosan structure determines nanoparticles preparation variables, as well as their final properties, such as size and zeta potential. The influence of these characteristics on the polymer performance in mucosal and transmucosal drug delivery was addressed in a recent review (Bonferoni et al. 2009).

The most important advantage claimed by those working with the methods of ionic gelation and polyelectrolyte complexation is the complete hydrophilic environment and the mild preparation conditions (Agnihotri et al. 2004). In fact, the avoidance of organic solvents or high shear forces makes encapsulation of labile drugs an easier task (Tiyaboonchai 2003) and, possibly for this reason, those two methods are the most widely used to produce chitosan nanoparticles. Importantly, through physical cross-linking mediated by an electrostatic interaction, instead of a chemical cross-linking with glutaraldehyde, those methods of chitosan nanoparticle production have improved cell viability and drug integrity (Janes et al. 2001, Agnihotri et al. 2004).

\section{Modified ionic gelation with radical polymerisation}

This method is derived from ionic gelation, but introduces a modification, because chitosan gelation occurs concomitantly with the polymerisation of acrylic acid monomers. The first step occurs at room temperature and consists in stirring an aqueous monomer solution of acrylic or 
methacrylic acid with an aqueous solution of oppositely charged chitosan. In some cases, polyethylene glycol (PEG) or polyether (polethylene glycol-polypropylene glycol-polyethylene glycol) are also added to the reaction medium, either separately into the monomer solution or following mixing with chitosan. As depicted in Figure 6, the opposite charges of chitosan and acrylic or methacrylic acid lead to an ionic interaction, while radical polymerisation of the latter is initiated by the addition of potassium persulfate. This reaction takes place under a nitrogen stream and the temperature is usually raised to $60-70{ }^{\circ} \mathrm{C}$. The polymerisation reaction lasts for approximately $6 \mathrm{~h}$, after which the formed suspension of nanoparticles is allowed to settle overnight. Finally, unreacted monomers are removed by dialysis or subsequent washes of the formed particles with distilled water. Nanoparticles obtained by this method have been used to administer insulin, bovine serum albumin and silk peptide through the oral route (Hu et al. 2002, Sajeesh and Sharma 2006a, Sajeesh and Sharma 2006b).

Increasing chitosan molecular weight was shown to induce an increase in the molecular weight of polyacrylic acid present in the nanoparticles, indicating the occurrence of a template polymerization of acrylic acid in the chitosan solution. Parameters such as chitosan/acrylic monomers ratio and polymers concentration have been found to strongly affect the physicochemical characteristics of the nanoparticles properties. More specifically, it was described that the zeta potential increases with the increase in chitosan/acrylic acid ratio, and nanoparticles size was smaller for the lowest polymer ratio (1/1) (Hu et al. 2002).

\section{Desolvation}

The method of desolvation is also frequently referred to as simple coacervation or phase separation and involves a macromolecular aggregation brought about by partial desolvation of fully solvated molecules (Kissel et al. 2006). The use of desolvating agents to produce chitosan particles was reported for the first time for the preparation of micron-sized carriers (Berthold et al. 1996) but, nowadays, this procedure is frequently applied to the production of chitosan 
nanoparticles. Substances such as sodium sulfate (Mao et al. 2001, Borges et al. 2005, Atyabi et al. 2009) and non-solvents miscible with water, like acetone (Agnihotri and Aminabhavi 2007), have been proposed as precipitating agents, although the former has been used more frequently. The preparation of chitosan nanoparticles by this method is very simple and mild as it involves the dropwise addition of the solvent competing agent of greater hydrophilicity (e.g. sodium sulfate) into a previously formed chitosan solution (Figure 7). As the salt enters in contact with the aqueous environment of chitosan solution, a progressive elimination of solvation water surrounding chitosan occurs as a consequence of the higher affinity of water for the salt. Eventually, this process leads to the polymer insolubilisation and its consequent precipitation (Alonso 1996, Janes et al. 2001, Poncelet 2005). This effect is observed because water-salt interactions are more favourable than those occurring between the water and the polymer, inducing the partial desolvation of chitosan. This, in turn, leads to increased interactions between chitosan molecules, forming the nanocarriers (Kissel et al. 2006).

It is very frequent to include a stabiliser such as polysorbate 80 in the preparation medium, to stabilise the nanoparticle suspension. A subsequent process of cross-linking, for instance with glutaraldehyde, has been described, in order to harden the nanoparticles (Alonso 1996). Factors such as chitosan molecular weight, chitosan concentration, amount of desolvating agent and stirring rate have been found to strongly affect the final characteristics of nanoparticles. Therefore, it is necessary to undergo an optimisation of these parameters. In addition, a correlation was identified between the amount of sulphate ions needed and chitosan properties, like the molecular weight and the deacetylation degree (Borges et al. 2005).

\section{Conclusion}

Several methods have been developed to produce chitosan nanoparticles, since those have been shown to offer attractive advantages as drug delivery carriers. The different production methods that were described might result in nanoparticles with distinct properties, including size 
and charge, which can affect their targeting ability, as well as their capacity for drug encapsulation. However, in addition to the preparation method, the final properties of nanoparticles also depend on several other parameters like secondary materials composing the matrix, material concentrations/ratios and reaction time, amongst others. In addition, the available studies report the use of distinct chitosans (molecular weight, deacetylation degree, etc.) and varied model/therapeutic molecules. Therefore, in this review, it was not possible to compare between the existing methods and conclude on those producing chitosan nanocarriers suitable for specific applications, although this would be extremely useful.

Some of the available methods involve long-lasting and, sometimes, somewhat aggressive procedures that limit their application. As a result, some are only reported in a few studies and, in such cases, many of the variables that might affect the final nanoparticle characteristics have not been thoroughly studied. In contrast, the methods that follow very mild procedures involving only hydrophilic environment (mainly polyelectrolyte complexation and ionic gelation), are being adopted by the majority of researchers working in the area. In fact, the day-by-day increasing amount of work that is based on the application of chitosan nanoparticles has been raising the expectation that, in a near future, a chitosan-based nanoparticulate formulation will reach the market. As a first step of acceptation of chitosan as excipient, the European Pharmacopeia already includes a monograph for chitosan hydrochloride salt and it is known that a monograph is in preparation for inclusion in the US Pharmacopeia.

\section{Declaration of interest}

Financial support from the Portuguese Foundation for Science and Technology (Project PTDC/SAU-FCF/100291/2008), QREN I\&DT Portugal (Project 13380 - MICALA) and IBB/CBME, LA is acknowledged. 


\section{References}

Agnihotri SA, Aminabhavi TM. 2007. Chitosan nanoparticles for prolonged delivery of timolol maleate. Drug Develop Ind Pharm, 33, 1254-1262.

Agnihotri SA, Mallikarjuna NN, Aminabhavi TM. 2004. Recent advances in chitosan-based micro-and nanoparticles in drug delivery. J Control Rel, 100, 5-28.

Agrawal P, Strijkers GJ, Nicolay K. 2010. Chitosan-based systems for molecular imaging. Adv Drug Deliv Rev, 62, 42-58.

Al-Qadi S, Grenha A, Remuñán-López C. 2011a. Chitosan and its derivatives as nanocarriers for siRNA delivery. J Drug Deliv Sci Technol, (accepted).

Al-Qadi , Grenha A, Carrión-Recio D, Seijo B, Remuñán-López C. 2011b. Microencapsulated chitosan nanoparticles for pulmonary protein delivery: In vivo evaluation of insulin-loaded formulations . J Control Release, doi:10.1016/j.jconrel.2011.08.008.

Alonso MJ. (1996). Nanoparticulate drug carrier technology. In: Cohen S and Bernstein H, eds. Microparticulate systems for the delivery of proteins and vaccines. New York: Marcel Dekker, 203-242.

Alonso MJ. 2004. Nanomedicines for overcoming biological barriers. Biomed Pharmacother, 58, 168-172.

Alonso-Sande M, Cuña M, Remuñán-López C, Teijeiro-Osorio D, Alonso-Lebrero JL, Alonso MJ. 2006. Formation of new glucomannan-chitosan nanoparticles and study of their ability to associate and deliver proteins. Macromolecules, 39, 4152-4158. 
Andrade F, Antunes F, Nascimento AV, da Silva SB, das Neves J, Ferreira D, Sarmento B. 2011. Chitosan formulations as carriers for therapeutic proteins. Curr Drug Discov Technol, 8, 157-172.

Anto SM, Kannan C, Kumar KS, Kumar SV, Suganeshwari M. 2011. Formulation of 5fluorouracil loaded chitosan nanoparticles by emulsion droplet coalescence method for cancer therapy. Int J Pharm Biol Arch, 2, 926-931.

Atyabi F, Talaie F, Dinarvand R. 2009. Thiolated chitosan nanoparticles as an oral delivery system for amikacin: in vitro and ex vivo evaluations. J Nanosci Nanotechnol, 9, 4593-4603.

Avadi MR, Sadeghi AMM. Mohammadpour N, Abedin S, Atyabi F, Dinarvand R, Rafiee-Tehrani M. 2010. Preparation and characterization of insulin nanoparticles using chitosan and arabic gum with ionic gelation method. Nanomed Nanotechnol Biol Med, 6, 58-63.

Azizi E, Namazi A, Haririan I, Fouladdel S, Khoshayand MR, Shotorbani PY. 2010. Release profile and stability evaluation of optimized chitosan/alginate nanoparticles as EGFR antisense vector. Int J Nanomed, 5, 455-461.

Baldrick P. 2010. The safety of chitosan as pharmaceutical excipient. Regul Toxicol Pharmacol, 56, 290-299.

Banerjee T, Mitra S, Singh AK, Sharma RK, Maitra A. 2002. Preparation, characterization and biodistribution of ultrafine chitosan nanoparticles. Int J Pharm, 243, 93-105.

Bayat A, Larijani B, Ahmadian S, Junginger HE, Rafiee-Tehrani M. 2008. Preparation and characterization of insulin nanoparticles using chitosan and its quaternized derivatives. Nanomed Nanotechnol Biol Med, 4, 115-120. 
Berthold A, Cremer K, Kreuter J. 1996. Preparation and characterisation of chitosan microspheres as drug carrier for prednisolone sodium phosphate as model for anti-inflammatory drugs. J Control Rel, 39, 17-25.

Bhattarai N, Gunn J, Zhang M. 2010. Chitosan-based hydrogels for controlled, localized drug delivery. Adv Drug Deliv Rev, 62, 83-99.

Bisht S, Maitra A. 2009. Dextran-doxorubicin/chitosan nanoparticles for solid tumor therapy. Wiley Interdiscip Rev Nanomed Nanobiotechnol, 1, 415-25.

Blau S, Jubeh TT, Haupt SM, Rubinstein A. 2000. Drug targeting by surface cationization. Crit Rev Ther Drug Carr Syst, 17, 425-465.

Bodmeier R, Chen HG, Paeratakul A. 1989. A novel approach to the oral delivery of micro- or nanoparticles. Pharm Res, 6, 413-417.

Bonferoni MC, Sandri G, Rossi S, Ferrari F, Caramella C. 2009. Chitosan and its salts for mucosal and transmucosal delivery. Expert Opin Drug Deliv, 6, 923-939.

Borges O, Borchard G, Verhoef JC, Sousa A, Junginger HE. 2005. Preparation of coated nanoparticles for a new mucosal vaccine delivery systems. Int J Pharm, 229, 155-166.

Bowman K, Leong KW. 2006. Chitosan nanoparticles for oral drug and gene delivery. Int J Nanomed, 1, 117-128.

Calvo P, Remuñan-Lopez C, Vila-Jato JL, Alonso MJ. 1997. Novel hydrophilic chitosanpolyethylene oxide nanoparticles as protein carriers. J Appl Polym Sci, 63, 125-132.

Canelas DA, Herlihy KP, DeSimone JM, 2009. Top-down particle fabrication: control of size and shape for diagnostic imaging and drug delivery. WIRE Nanomed Nanobiotechnol, 1, 391-404. 
Carvalho ELS, Grenha A, Remuñán-López C, Alonso MJ, Seijo B. 2009. Mucosal delivery of liposome-chitosan nanoparticle complexes. Methods Enzymol, 465, 289-312.

Chan H-K, Kwok PCL. 2011. Production methods for nanodrug particles using the bottom-up approach. Adv Drug Deliv Rev, 63, 406-416.

Chaudury A, Das S. 2011. Recent advancement of chitosan-based nanoparticles for oral controlled delivery of insulin and other therapeutic agents. AAPS PharmSciTech, 12, 10-20.

Chiellini F, Piras AM, Errico C, Chiellini E. 2008. Micro/nanostructured polymeric systems for biomedical and pharmaceutical applications. Nanomedicine, 3, 367-393.

Csaba N, Garcia-Fuentes M, Alonso MJ. 2006. The performance of nanocarriers for transmucosal drug delivery. Expert Opin Drug Deliv, 3, 463-478.

Cuña M, Alonso-Sande M, Remuñan-Lopez C, Pivel JP, Alonso-Lebrero JL, Alonso MJ. 2006. Development of phosphorylated glucomannan-coated chitosan nanoparticles as nanocarriers for protein delivery. J Nanosci Nanotechnol, 6, 2887-2895.

das Neves J, Bahia M, Amiji MM, Sarmento B. 2011. Mucoadhesive nanomedicines: characterization and modulation of mucoadhesion at the nanoscale. Expert Opin Drug Delivery, $8,1085-1104$.

de Campos A, Sanchez A, Alonso MJ. 2001. Chitosan nanoparticles: a new vehicle for the improvement of the delivery of drugs to the ocular surface. Application to cyclosporin A. Int $J$ Pharm, 224,159-168.

de la Fuente M, Csaba N, Garcia-Fuentes M, Alonso MJ. 2008a. Nanoparticles as protein and gene carriers to mucosal surfaces. Nanomedicine, 3, 845-857. 
de la Fuente M, Seijo B, Alonso MJ. 2008b. Novel hyaluronic acid-chitosan nanoparticles for ocular gene therapy. ", Invest Ophtalmol Vis Sci, 49, 2016-2024.

Desai MP, Labhasetwar V, Amidon GL, Levy RJ. 1996. Gastrointestinal uptake of biodegradable microparticles: effect of particle size. Pharm Res, 13, 1838-1845.

Du J, Sun R, Zhang S, Govender T, Zhang LF, Xiong CD, Peng YX. 2004. Novel polyelectrolyte carboxymethyl konjac glucomannan-chitosan nanoparticles for drug delivery. Macromol Rapid Commun, 25, 954-958.

Duceppe N, Tabrizian M. 2010. Advances in using chitosan-based nanoparticles for in vitro and in vivo drug and gene delivery. Expert Opin Drug Deliv, 7, 1191-1207.

El-Shabouri MH. 2002. Positively charged nanoparticles for improving the oral bioavailability of cyclosporin-A. Int J Pharm, 249, 101-108.

Elsayed A, Al-Remawi M, Qinna N, Farouk A, Al-sou'od K, Badwan A. 2011. Chitosan-sodium lauryl sulfate nanoparticles as a carrier system for the in vivo delivery of oral insulin. AAPS PharmsciTech, 12, 958-964.

Erbacher P, Zou S, Bettinger T, Steffan A-M, Remy J-S. 1998. Chitosan-based vector/DNA complexes for gene delivery: biophysical characteristics and transfection ability. Pharm Res, 15, $1332-1339$.

Fan W, Yan W, Xu Z, Ni H. 2012. Formation mechanism of monodisperse, low molecular weight chitosan nanoparticles by ionic gelation technique. Colloid Surf B-Biointerfaces, 90, 21-27.

FDA, (2005). Guidance for industry - Ninclinical studies for the safety evaluation of pharmaceutical excipients. [Online] Available at: 


\section{http://www.fda.gov/downloads/Drugs/GuidanceComplianceRegulatorylnformation/Guidances/uc}

m079250.pdf Accessed on 8 December 2011.

Goycoolea F, Lollo G, Remuñán-López C, Quaglia F, Alonso MJ. 2009. Chitosan-alginate blended nanoparticles as carriers for the transmucosal delivery of macromolecules.

Biomacromolecules, 10, 1736-1743.

Grabnar P, Kristl J. 2010. Physicochemical characterization of protein-loaded pectin-chitosan nanoparticles prepared by polyelectrolyte complexation. Pharmazie, 65, 851-852.

Grenha A, Al-Qadi S, Seijo B, Remuñán-Lopez C. 2010a. The potential of chitosan for pulmonary drug delivery. J Drug Deliv Sci Technol, 20, 33-43.

Grenha A, Gomes ME, Rodrigues M, Santo VE, Mano JF, Neves NM, Reis RL. 2010b. Development of new chitosan/carrageenan nanoparticles for drug delivery applications. $J$ Biomed Mater Res, 92A, 1265-1272.

Grenha A, Seijo B, Remuñan-Lopez C. 2005. Microencapsulated chitosan nanoparticles for lung protein delivery. Eur J Pharm Sci, 25, 427-437.

Hirano S, Seino H, Akiyama Y, Nonaka I. 1988. Biocompatibility of chitosan by oral and intravenous administrations. Polym Mater Sci Eng, 59, 897-901.

Hu Y, Jiang X, Ding Y, Ge H, Yuan Y, Yang C. 2002. Synthesis and characterization of chitosanpoly(acrylic acid) nanoparticles. Biomaterials, 23, 3193-3201.

Huang M, Khor E,Lim L-Y. 2004. Uptake and cytotoxicity of chitosan molecules and nanoparticles: effects of molecular weight and degree of deacetylation. Pharm Res, 21, 344-353. 
Ichikawa H, Tokumitsu H, Miyamoto M, Fukumori Y. (2006). Nanoparticles for neutron capture therapy of cancer. In: Kumar C, ed. Nanomaterials for cancer therapy. Weinheim: Wiley-VCH, $87-121$.

Janes KA, Calvo P, Alonso MJ. 2001. Polysaccharide colloidal particles as delivery systems for macromolecules. Adv Drug Deliv Rev, 47, 83-97.

Jani P, Halbert GW, Langridge J, Florence AT. 1990. Nanoparticle uptake by the rat gastrointestinal mucosa: quantitation and particle size dependency. J Pharm Pharmacol, 42, 821-826.

Kaihara S, Suzuki Y, Fujimoto K. 2011. In situ synthesis of polysaccharide nanoparticles via polyion complex of carboxymethyl cellulose and chitosan. Colloid Surf B - Biointerfaces, 85, 343348.

Kean T, Thanou M. 2010. Biodegradation, biodistribution and toxicity of chitosan. Adv Drug Deliv Rev, 62, 3-11.

Keawchaoon L. Yoksan R. 2011. Preparation, characterization and in vitro release study of carvacrol-loaded chitosan nanoparticles. Colloid Surf B - Biointerfaces, 84, 163-171.

Kissel T, Maretscheck S, Packhäuser C, Schnieders J, Seidel N. (2006). Microencapsulation techniques for parenteral depot systems and their application in the pharmaceutical industry. In: Benita S, ed. Microencapsulation: methods and industrial applications. New York: Taylor \& Francis, 99-122.

Lehr CM, Bouwstra JA, Schacht EH, Junginger HE. 1992. In vitro evaluation of mucoadhesive properties of chitosan and some other natural polymers. Int J Pharm, 78, 43-48. 
Lin Y-H, Mi F-L, Chen C-T, Chang W-C, Peng S-F, Liang H-F, Sung H-W. 2007. Preparation and characterisation of nanoparticles shelled with chitosan for oral insulin delivery.

Biomacromolecules, 8, 146-152.

Lin Y-H, Sonaje K, Lin KM, Juang J-H, Mi F-L, Yang H-W, Sung H-W. 2008. Multi-ioncrosslinked nanoparticles with $\mathrm{pH}$-responsive characteristics for oral delivery of protein drugs. $\mathrm{J}$ Control Rel, 132, 141-149.

Liu H, Chen B, Mao Z, Gao C. 2007a. Chitosan nanoparticles for loading of toothpaste actives and adhesion on tooth analogs. J Appl Polym Sci, 106, 4248-4256.

Liu Z, Jiao Y, Liu F, Zhang Z. 2007b. Heparin/chitosan nanoparticle carriers prepared by polyelectrolyte complexation. J Biomed Mater Res, 83A, 806-812.

Luppi B, Bigucci F, Cerchiara T, Zecchi V. 2010. Chitosan-based hydrogels for nasal drug delivery: from inserts to nanoparticles. Expert Opin Drug Deliv, 7, 811-828.

Ma Z, Yeoh HH, Lim L-Y. 2002. Formulation pH modulates the interaction of insulin with chitosan nanoparticles. J Pharm Sci, 91, 1396-1404.

Malam Y, Lim EJ, Seifalian AM. 2011. Current trends in the application of nanoparticles in drug delivery. Current Med Chem, 18, 1067-1078.

Manchanda R, Nimesh R. 2010. Controlled size chitosan nanoparticles as an efficient, biocompatible oligonucleotides delivery system. J Appl Polym Sci, 118, 2071-2077.

Mao H-Q, Roy K, Troung-Le VL, Janes KA, Lin KY, Wang Y, August JT, Leong KW. 2001. Chitosan-DNA nanoparticles as gene carriers: synthesis, characterization and transfection efficiency. J Control Rel, 70, 399-421. 
Maurya SK, Pathak K, Bali V. 2010. Therapeutic potential of mucoadhesive drug delivery systems - an updated patent review. Recent Patents Drug Deliv Formul, 4, 256-265.

Mitra S, Gaur U, Ghosh PC, Maitra AN. 2001. Tumor targeted delivery of encapsulated dextrandoxorubicin conjugate using chitosan nanoparticles as carriers. J Control Rel, 74, 317-323.

Nagpal K, Singh SK, Mishra DN. 2010. Chitosan nanoparticles: a promising system in novel drug delivery. Chem Pharm Bull (Tokyo), 58, 1423-1430.

Niwa T, Takeuchi H, Hino T, Kunou N, Kawashima Y. 1993. Preparation of biodegradable nanospheres of water soluble and insoluble drugs with D,L-lactide/glycolide copolymer by a novel spontaneous emulsification solvent diffusion method, and the drug release behavior. $J$ Control Rel, 2, 89-98.

Ohya Y, Shiratani M, Kobayashi H, Ouchi T. 1994. Release behaviour of 5-fluorouracil from chitosan-gel nanospheres immobilizing 5-fluorouracil coated with polysaccharides and their cell specific cytotoxicity. Pure Appl Chem, A31, 629-642.

Oyarzun-Ampuero FA, Brea J, Loza MI, Torres D, Alonso MJ. 2009. Chitosan-hyaluronic acid nanoparticles loaded with heparin for the treatment of asthma. Int J Pharm, 381, 122-129.

Park JH, Saravanakumar G, Kim K, Kwon IC. 2010. Targeted delivery of low molecular drugs using chitosan and its derivatives. Adv Drug Deliv Rev, 62, 28-41.

Pileni MP. 2006. Reverse micelles used as templates: a new understanding in nanocrystal growth. J Experim Nanosci, 1, 13-27.

Poncelet D. (2005). Microencapsulation: fundamentals, methods and applications. In: Blitz JP, Gun'ko VM, eds. Surface chemistry in biomedical and environmental science. Dordrecht: Springer, 23-34. 
Posadas I, Guerra FJ, Ceña V. 2010. Nonviral vectors for the delivery of small interfering RNAs to the CNS. Nanomedicine (Lond), 5, 1219-36.

Prabaharan M, Mano JF. 2005. Chitosan-based particles as controlled drug delivery systems. Drug Deliv, 12, 41-57.

Rejinold N, Muthunarayanan M, Muthuchelian K, Chennazhi K, Nair S, Jayakumar R. 2011. Saponin-loaded chitosan nanoparticles and their cytotoxicity to cancer cell lines in vitro. Carb Polym, 84, 407-416.

Sajeesh S, Sharma CP. 2006a. Cyclodextrin-insulin complex encapsulated polymethacrylic acid based nanoparticles for oral insulin delivery. Int J Pharm, 325, 147-154.

Sajeesh S, Sharma CP. 2006b. Novel pH responsive polymethacrylic acid-chitosan-polyethylene glycol nanoparticles for oral peptide delivery. J Biomed Mater Res, 76B, 298-305.

Sarmento B, Martins S, Ribeiro A, Veiga F, Neufeld R, Ferreira D. 2006a. Development and comparison of different nanoparticulate polyelectrolyte complexes as insulin carriers. Int $J$ Peptide Res Ther, 12, 131-138.

Sarmento B, Ribeiro A, Veiga F, Ferreira D. 2006b. Development and characterization of new insulin containing polysaccharide nanoparticles. Colloid Surf B - Biointerfaces, 53, 193-202.

Sarmento, Ribeiro A, Veiga F, Ferreira D, Neufeld R. 2007. Oral bioavailability of insulin contained in polysaccharide nanoparticles. Biomacromolecules, 8, 3054-3060.

Schipper NGM, Vårum KM, Artursson P. 1996. Chitosans as absorption enhancers for poorly absorbable drugs. 1: Influence of molecular weight and degree of acetylation on drug transport across human intestinal epithelial (Caco-2) cells. Pharm Res, 13, 1686-1692. 
Schipper NGM, Vårum KM, Stenberg P, Ocklind G, Lennernäs H, Artursson P. 1999. Chitosans as absorption enhancers of poorly absorbable drugs 3: influence of mucus on absorption enhancement. Eur J Pharm Sci, 8, 335-343.

Silva GA, Coutinho OP, Ducheyne P, Shapiro I M, Reis RL. 2007. Starch-based microparticles as carriers for the release of active platelet-derived growth factor. Tissue Eng, 13, 1259-1268.

Songjiang Z, Lixiang W. 2009. Amyloid-Beta associated with chitosan nano-carrier has favorable immunogenicity and permeates the BBB. AAPS PharmsciTech, 10, 900-905.

Takeuchi H, Yamamoto H, Kawashima Y. 2001. Mucoadhesive nanoparticulate systems for peptide drug delivery. Adv Drug Deliv Rev, 47, 39-54.

Tan ML, Choong PF, Dass CR. 2009. Cancer, chitosan nanoparticles and catalytic nucleic acids. J Pharm Pharmacol, 61, 3-12.

Tang Z-X, Qian J-Q, Xi L-E. 2007. Preparation of chitosan nanoparticles as carrier for immobilized enzyme. Appl Biochem Biotechnol, 136, 77-96.

Teijeiro-Osorio D, Remuñan-López C, Alonso MJ. 2009. New generation of hybrid poly/oligosaccharide nanoparticles as carriers for the nasal delivery of macromolecules. Biomacromolecules, 10, 243-249.

Terbojevich M, Muzzarelli RAA. (2009). Chitosan. In: Phillips GO, Williams P, eds. Handbook of hydrocolloids. Cambridge: Woodhead Publishing Ltd., 367-378.

Tian XX, Groves MJ. 1999. Formulation and biological activity of antineoplastic proteoglycans derived from Mycobacterium vaccae in chitosan nanoparticles. J Pharm Pharmacol, 51, 151157. 
Tiera MJ, Shi Q, Winnik FM, Fernandes JC. 2011. Polycation-based gene therapy: current knowledge and new perspectives. Curr Gene Ther, 11, 288-306.

Tiyaboonchai W. 2003. Chitosan nanoparticles: a promising system for drug delivery. Naresuan Univ J, 11, 51-66.

Tokumitsu H, Ichikawa H, Fukumori Y, Block LH. 1999a. Preparation of gadopentetic acidloaded chitosan microparticles for gadolinium neutron-capture therapy of cancer by a novel emulsion-droplet coalescence technique. Chem Pharm Bull, 47, 838-842.

Tokumitsu H, Ichikawa H, Fukumori Y. 1999b. Chitosan-gadopentetic acid complex nanoparticles for gadolinium neutron capture therapy of cancer: preparation by novel emulsiondroplet coalescence technique and characterization. Pharm Res, 16, 1830-1835.

Wang JJ, Zeng Z, Xiao R, Xie T, Zhou G, Zhan X, Wang S. 2011a. Recent advances of chitosan nanoparticles as drug carriers. Int J Nanomed, 6, 765-774.

Wang J, Byrne JD, Napier ME, DeSimone JM. 2011b. More effective nanomedicines through particle design. Small, 7, 1919-1931.

Wang X, Chi N, Tang X. 2008. Preparation of estradiol chitosan nanoparticles for improving nasal absorption and brain targeting. Eur J Pharm Biopharm, 70, 735-740.

Xu J, Dai W, Wang Z, Chen B, Li Z, Fan X. 2011. Intranasal vaccination with chitosan-DNA nanoparticles expressing Pneumococcal Surface Antigen A protects mice against nasopharyngeal colonization by Streptococcus pneumoniae. Clin Vaccine Immunol, 18, 75-81.

Xu Y, Du Y. 2003. Effect of molecular structure of chitosan on protein delivery properties of chitosan nanoparticles. Int J Pharm, 250, 215-226. 
Yang S, Lin F, Tsai H, Lin C, Chin H, Wong J, Shieh M. 2011. Alginate-folic acid-modified chitosan nanoparticles for photodynamic detection of intestinal neoplasms. Biomaterials, 32, 2174-2182.

Yeh MK, Cheng KM, Hu CS, Huang YC, Young JJ. 2011. Novel protein-loaded chondroitin sulfate-chitosan nanoparticles: Preparation and characterization. Acta Biomater, doi:10.1016/j.actbio.2011.06.026.

Zhang H, Oh M, Allen C, Kumacheva E. 2004. Monodisperse chitosan nanoparticles for mucosal drug delivery. Biomacromolecules, 5, 2461-2468.

Zheng C, Yan W, Li-Jun Z, Zhong-Qiu L. 2010. Nanocarriers: A general strategy for enhancement of oral bioavailability of poorly absorbed or pre-systemically metabolized drugs. Curr Drug Metab, 11, 197-207.

\section{Figure captions}

Figure 1 - Schematic representation of the method of emulsification and cross-linking.

Figure 2 - Schematic representation of the method of emulsion droplet coalescence.

Figure 3 - Schematic representation of the method of emulsion solvent diffusion.

Figure 4 - Schematic representation of the method of reverse micellisation.

Figure 5 - Schematic representation of the method of ionic gelation/polyelectrolyte complexation.

Figure 6 - Schematic representation of the method of modified ionic gelation with radical polymerisation.

Figure 7 - Schematic representation of the method of desolvation with sodium sulfate. 
Table 1. Methods used for the production of chitosan-based nanoparticles and composition of the carriers' matrix

\begin{tabular}{|c|c|c|}
\hline Production Method & Matrix composition & Reference \\
\hline $\begin{array}{l}\text { Emulsification and cross- } \\
\text { linking }\end{array}$ & Chitosan, glutaraldehyde & $\begin{array}{l}\text { (Ohya et al. 1994, } \\
\text { Songjiang and Lixiang } \\
\text { 2009) }\end{array}$ \\
\hline $\begin{array}{l}\text { Emulsion droplet } \\
\text { coalescence }\end{array}$ & Chitosan & $\begin{array}{l}\text { (Tokumitsu et al. 1999, } \\
\text { Anto et al. 2011) }\end{array}$ \\
\hline Emulsion solvent diffusion & Chitosan & (El-Shabouri 2002) \\
\hline Reverse micellisation & Chitosan, glutaraldehyde & $\begin{array}{l}\text { (Mitra et al. 2001, } \\
\text { Banerjee et al. 2002, } \\
\text { Tang et al. 2007, } \\
\text { Manchanda and Nimesh } \\
\text { 2010) }\end{array}$ \\
\hline Ionic gelation & Chitosan, tripolyphosphate & $\begin{array}{l}\text { (Calvo et al. 1997, de } \\
\text { Campos et al. 2001, Ma } \\
\text { et al. 2002, Wang et al. } \\
\text { 2008; Fan et al. 2012) }\end{array}$ \\
\hline $\begin{array}{l}\text { Polyelectrolyte } \\
\text { complexation }\end{array}$ & $\begin{array}{l}\text { Chitosan, alginate, arabic } \\
\text { gum, carboxymethyl } \\
\text { cellulose, carrageenan, } \\
\text { chondroitin sulfate, } \\
\text { cyclodextrins, dextran } \\
\text { sulfate, polyacrylic acid, } \\
\text { poly- } \gamma \text {-glutamic acid, insulin, } \\
\text { DNA }\end{array}$ & $\begin{array}{l}\text { (Erbacher et al. 1998, Hu } \\
\text { et al. 2002, Du et al. } \\
\text { 2004, Sarmento et al. } \\
\text { 2006a, Sarmento et al. } \\
\text { 2006b, Lin et al. 2007, } \\
\text { Bayat et al. 2008, Lin et } \\
\text { al. 2008, Teijeiro-Osorio } \\
\text { et al. 2009, Avadi et al. } \\
\text { 2010, Grenha et al. } \\
\text { 2010b, Kaihara et al. } \\
\text { 2011, Yeh et al. 2011) }\end{array}$ \\
\hline $\begin{array}{l}\text { Modified ionic gelation } \\
\text { with radical polymerisation }\end{array}$ & $\begin{array}{l}\text { Chitosan, acrylic acid, } \\
\text { methacrylic acid, } \\
\text { polyethylene glycol, } \\
\text { polyether }\end{array}$ & $\begin{array}{l}\text { (Hu et al. 2002, Sajeesh } \\
\text { and Sharma 2006a, } \\
\text { Sajeesh and Sharma } \\
\text { 2006b) }\end{array}$ \\
\hline Desolvation & Chitosan & $\begin{array}{l}\text { (Mao et al. 2001, Borges } \\
\text { et al. 2005, Agnihotri and } \\
\text { Aminabhavi 2007, } \\
\text { Atyabi et al. 2009) }\end{array}$ \\
\hline
\end{tabular}




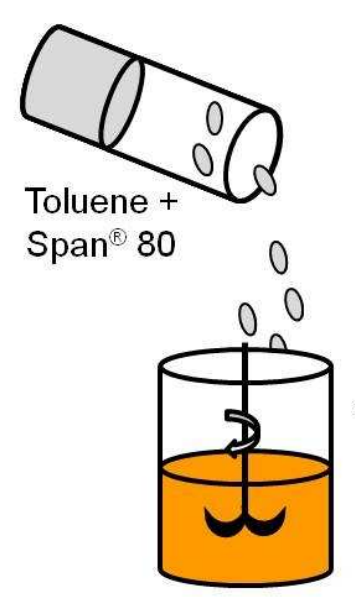

Chitosan (CS) +5 -fluorouracil

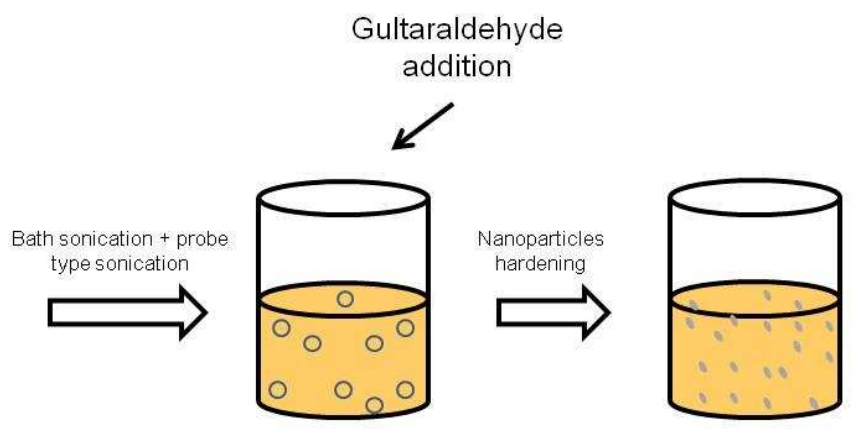

W/O emulsion (NP formation)
Cross-linked CS nanoparticles

Schematic representation of the method of emulsification and cross-linking. $177 \times 93 \mathrm{~mm}(150 \times 150$ DPI $)$ 


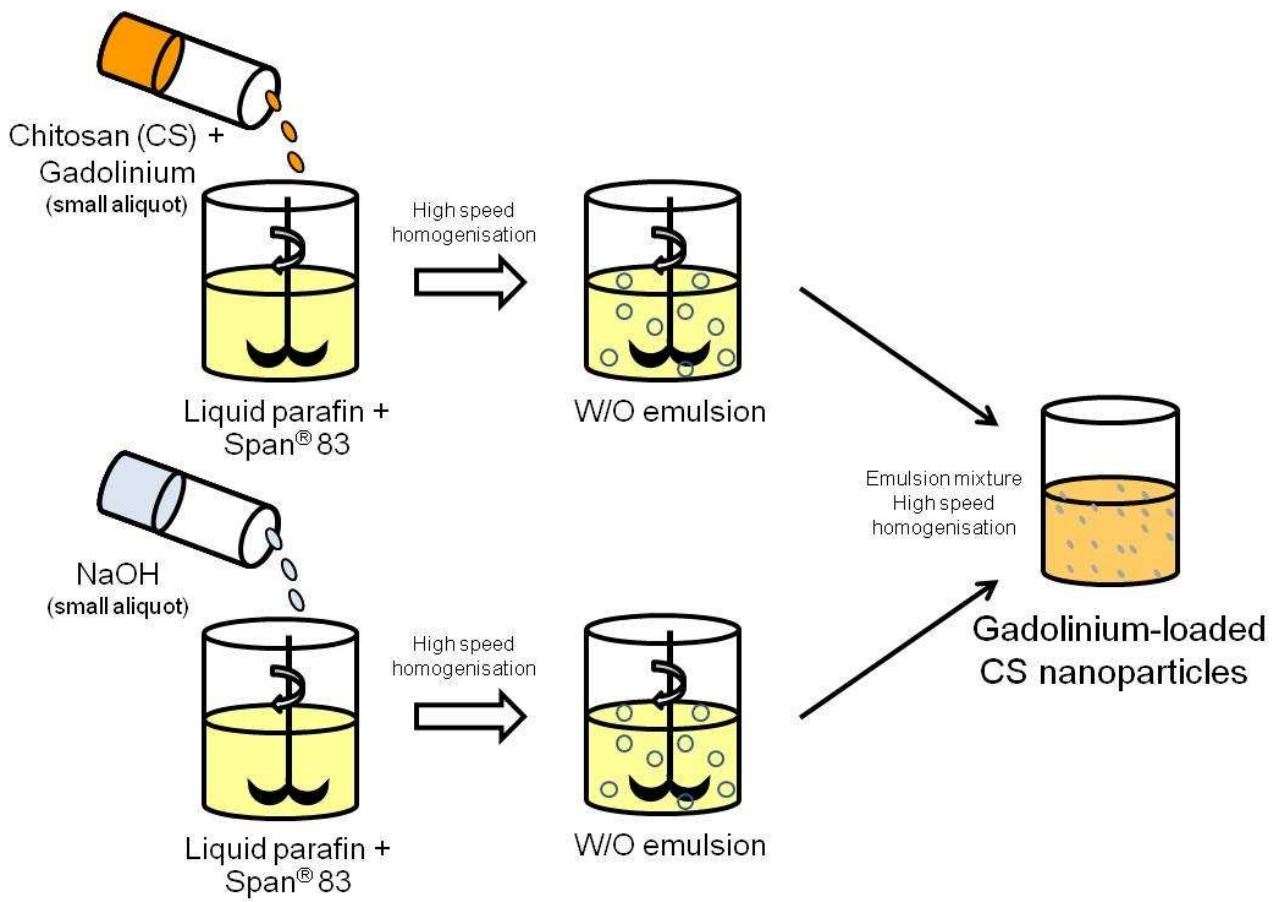

Schematic representation of the method of emulsion droplet coalescence. $180 \times 123 \mathrm{~mm}(150 \times 150 \mathrm{DPI})$ 


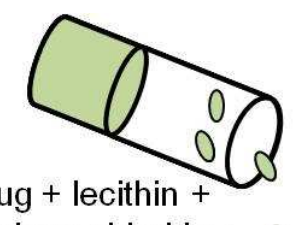

methylene chloride +0 acetone

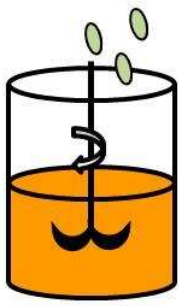

Chitosan (CS) + poloxamer

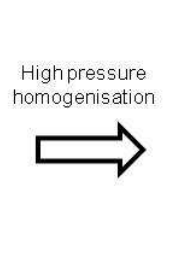

O/W emulsion
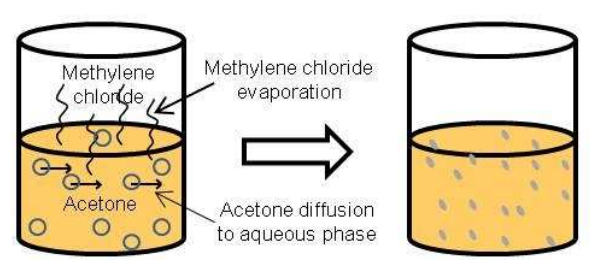

CS nanoparticles

Schematic representation of the method of emulsion solvent diffusion. $182 \times 91 \mathrm{~mm}(150 \times 150 \mathrm{DPI})$ 


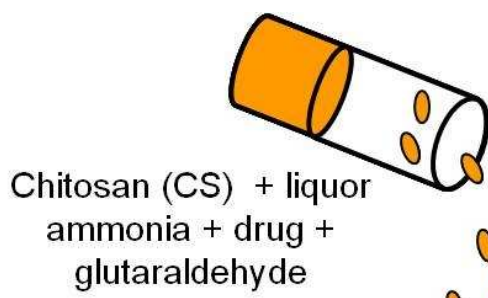

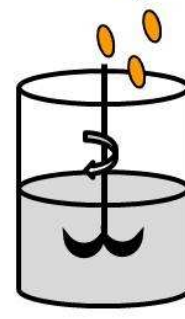

Sodium bis(ethyl hexyl) sulfosuccinate

+ n-hexane

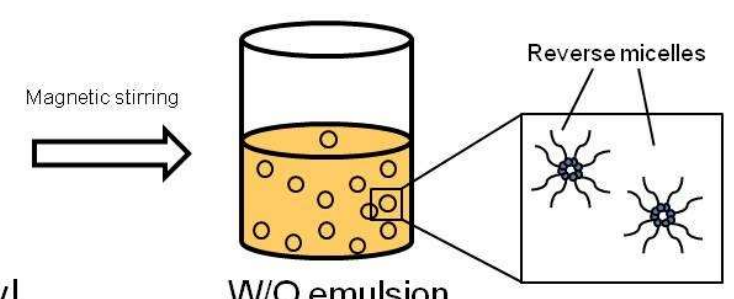

W/O emulsion (CS nanoparticles)

Schematic representation of the method of reverse micellisation. $178 \times 96 \mathrm{~mm}(150 \times 150 \mathrm{DPI})$ 


1
2
3
4
5
6
7
8
9
10
11
12
13
14
15
16
17
18
19
20
21
22
23
24
25
26
27
28
29
30
31
32
33
34
35
36
37
38
39
40
41
42
43
44
45
46
47
48
49
50
51
52
53
54
55
56
57
58
60
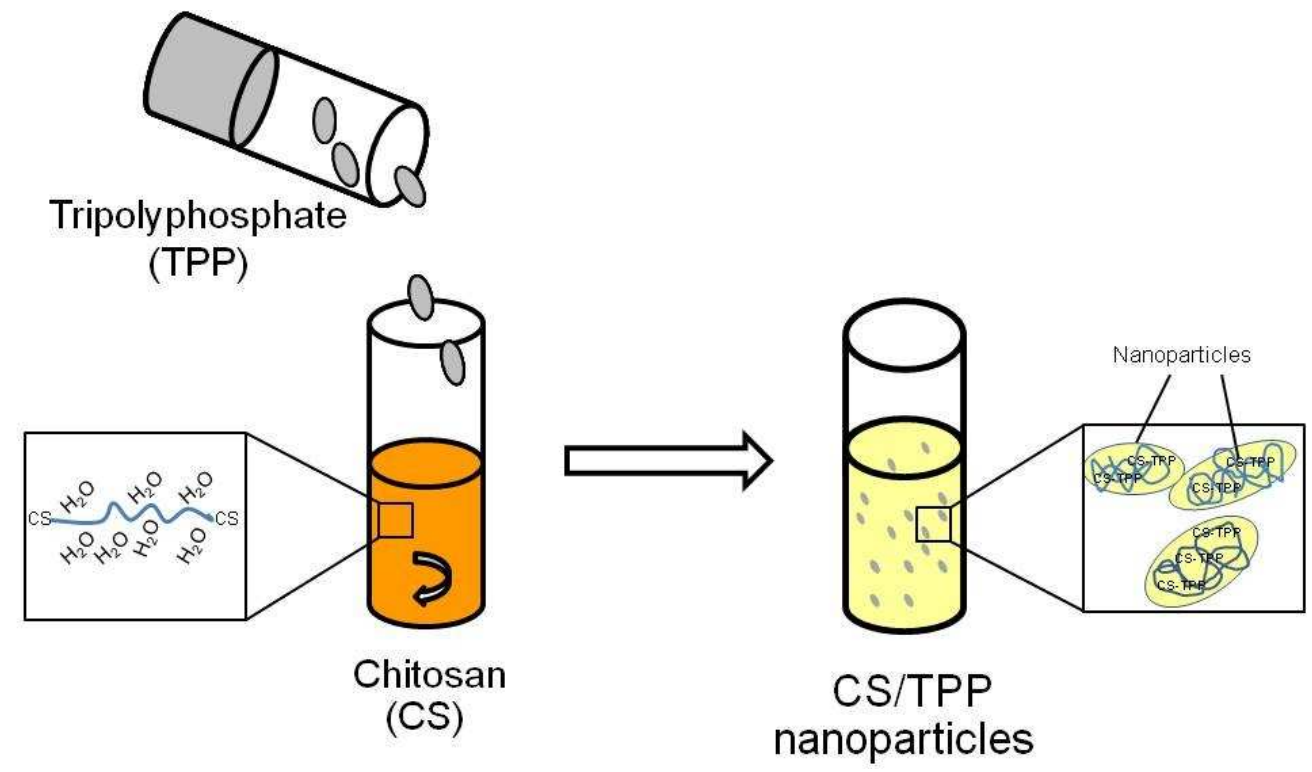

Schematic representation of the method of ionic gelation/polyelectrolyte complexation. $162 \times 94 \mathrm{~mm}(150 \times 150 \mathrm{DPI})$ 


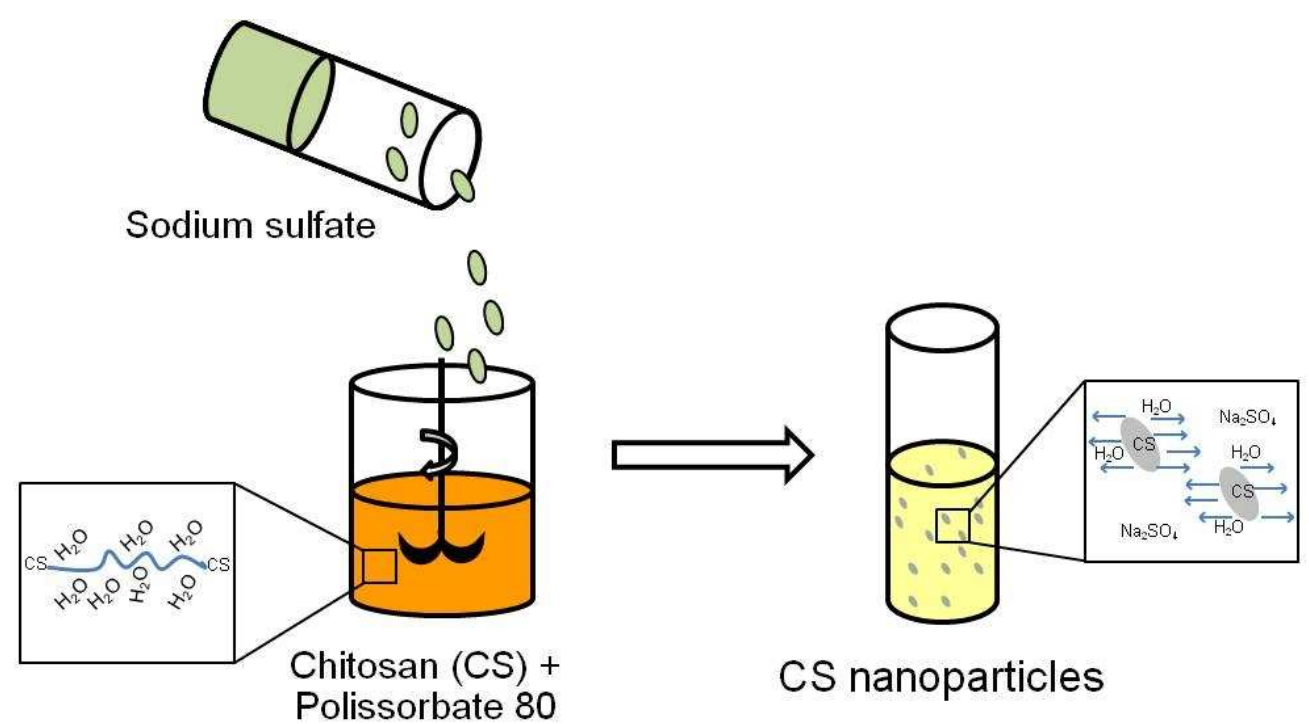

Schematic representation of the method of desolvation with sodium sulfate. $167 \times 91 \mathrm{~mm}(150 \times 150 \mathrm{DPI})$ 